\title{
Universiteit
}

Leiden

The Netherlands

\section{A FRET-based biosensor for NO detection}

Strianese, M.; De Martino, F.; Pavone, V.; Lombardi, A.; Canters, G.W.; Pellecchia, C.

\section{Citation}

Strianese, M., De Martino, F., Pavone, V., Lombardi, A., Canters, G. W., \& Pellecchia, C. (2010). A FRET-based biosensor for NO detection. Journal Of Inorganic Biochemistry, 104(6), 619-624. doi:10.1016/j.jinorgbio.2010.02.002

Version: $\quad$ Publisher's Version

License: $\quad$ Licensed under Article 25fa Copyright Act/Law (Amendment Taverne)

Downloaded from: https://hdl.handle.net/1887/3192254

Note: To cite this publication please use the final published version (if applicable). 


\title{
A FRET-based biosensor for NO detection
}

\author{
Maria Strianese ${ }^{\mathrm{a}}$, Franco De Martino ${ }^{\mathrm{a}}$, Vincenzo Pavone ${ }^{\mathrm{c}}$, Angela Lombardi ${ }^{\mathrm{c}}$, \\ Gerard W. Canters ${ }^{\mathrm{b}, *}$, Claudio Pellecchia ${ }^{\mathrm{a}, *}$ \\ a Dipartimento di Chimica, Università di Salerno, via Ponte Don Melillo, Fisciano (Sa), I84084 Italy \\ ${ }^{\mathrm{b}}$ Leiden Institute of Chemistry, Leiden University, Einsteinweg 55, 2333 CC Leiden, The Netherlands \\ c Dipartimento di Chimica, Università di Napoli, Via Cintia 45, I-80126 Napoli, Italy
}

\section{A R T I C L E I N F O}

\section{Article history:}

Received 30 April 2009

Received in revised form 17 February 2010

Accepted 19 February 2010

Available online 2 March 2010

\section{Keywords:}

Heme proteins

Biosensors

Fluorescence

FRET

Sol-gel processes

\begin{abstract}
A B S T R A C T
In this paper we explore the use of fluorescently labeled cytochrome $c$ peroxidase (CcP) from baker's yeast for monitoring nitric oxide (NO) down to the sub-micromolar level, by means of a FRET (Förster Resonance Energy Transfer) mechanism. The binding affinity constant $\left(K_{\mathrm{d}}\right)$ for the NO binding to CcP was determined to be $10 \pm 1.5 \mu \mathrm{M}$. The rate of NO dissociation from the CcP $\left(k_{\text {off }}\right)$ and the second order rate constant for the NO association $\left(k_{\text {on }}\right.$ ) were found to be $0.22 \pm 0.08 \mathrm{~min}^{-1}$ and $0.024 \pm 0.002 \mu \mathrm{M}^{-1} \mathrm{~min}^{-1}$ respectively. The immobilization of fluorescently labeled CcP into a polymeric matrix for use in a solid state NO sensing device was also explored. The results provide proof-of-principle that labeled CcP can be successfully implemented in a fast, simple, quantitative and sensitive NO sensing device.
\end{abstract}

(c) 2010 Elsevier Inc. All rights reserved.

\section{Introduction}

In 1992, nitric oxide (NO) was selected by the journal "Science" as "Molecule of the year" [1]. This reflected the discovery that NO plays a crucial role in a variety of biological processes [2]. At low concentrations, NO regulates vasodilation in the circulatory system and serves as a messenger in the immune and nervous systems [3]. At micromolar concentrations NO can lead to carcinogenic and neurodegenerative disorders [4,5]. Furthermore NO is a ubiquitous by-product of hightemperature combustion [6] and one of the hazardous exhaust gases generated by motor vehicles [7]. Emissions of these gases cause local and global environmental problems such as acid rain, greenhouse effects, destruction of the ozone layer and air pollution.

With such diverse interests in NO, there is a pressing need for methods to detect NO in both aqueous and gaseous media [8]. Currently available technologies to monitor NO levels are based among others on chemiluminescent instrumentation [9], amperometry [10], EPR spectroscopy [11], colorimetric assays or porphyrin based fluorescent compounds [12]. While each technique has its own advantages $[8,13,14]$ there remains a need for simple to implement, cheap NO sensors that can be used repeatedly [15].

With fluorescence-based methods one may, in principle, be able to avoid some of the limitations of existing methodologies. In 1998 the first

\footnotetext{
* Corresponding authors. Pellecchia is to be contacted at Tel.: +39 089 969576; fax: +39 089 969603. Canters, Tel.: +31 71527 4256; fax: +31 715274349 .

E-mail addresses: canters@chem.leidenuniv.nl (G.W. Canters), cpellecchia@unisa.it (C. Pellecchia).
}

example of a fluorescence-based system for sensing NO was reported. The method makes use of cytochrome $c^{\prime}$ immobilized on gold nanobeads as sensing material. The sensor operates in a reversible way and the reported detection limit was $20 \mu \mathrm{M}$ [16]. One year later the same group improved the performance of this sensor by employing fluorescent ratiometric measurements. The improved sensor consisted of fluorescently labeled cytochrome $c^{\prime}$ incorporated within polystyrene nanobeads labeled with a fluorescent reference dye, whose emission allowed ratiometric (two wavelengths) measurements. Compared to the previous construct, the detection limit of the sensor was lowered to $8 \mu \mathrm{M}$ [17]. A relevant application of this NO sensing system is the measurement of NO produced by macrophages $[17,18]$. The system relies on a FRET-based (Förster Resonance Energy Transfer) mechanism by which the dye fluorescence is attenuated as the protein binds NO. The observed fluorescence changes were ascribed [16] to conformational alterations in cyt $c^{\prime}$ induced by NO binding. This type of sensor is commonly referred to as a "turn-off" sensor since it exploits the quenching of the dye fluorescence upon analyte binding.

FRET-based ratiometric fiber-optic NO sensors have been prepared with the heme domain of soluble guanylate cyclase [18]. They also belong to the family of the "turn-off" sensors, and function in a manner analogous to the biosensors prepared with cytochrome $c^{\prime}$. With a genetically encoded fluorescent indicator, NOA-1, physiological nanomolar dynamics of NO in single living cells have been reported as detectable [19]. The sensing system is suitable for monitoring NO release by endothelial cells, neurons and macrophages [19].

Fluorescent detection of NO has also been achieved with the commercially available organic molecules o-diaminonaphthalene 
(DAN) and the o-diamino-fluoresceins (DAFs) [13]. Their fluorescence depends on the formation of a triazole species by oxidized NO products (e.g. $\mathrm{N}_{2} \mathrm{O}_{3}$ ), which means that the $\mathrm{NO}$ is detected in an indirect way. The theoretical detection limit for these constructs amounts to $10 \mathrm{nM}$ [13], although it remains to be established what the detection limit will be under practical circumstances like in a cell supernatant.

Recently we and other groups have reported that it is possible to detect oxygen using a type-3 copper protein labeled with a fluorescent dye, by means of a FRET mechanism [20,21]. To design an NO sensor along the same principles, we extended the FRET-based approach to cytochrome c peroxidase from baker's yeast (CcP), making use of the fact that its optical characteristics are different in the presence and absence of NO. CcP is a soluble 34-kDa heme protein, located in the intermembrane space of yeast mitochondria, which is structurally, spectroscopically and functionally well characterized [22,23]. Resting state $\mathrm{CcP}\left(\mathrm{Fe}^{\mathrm{III}}\right)$ contains a noncovalently bound heme with a five-coordinate, high spin iron $(S=5 / 2)$. The sixth coordination position is vacant, allowing ligands like NO to bind [22].

The overall properties of CCP make it an attractive candidate for developing a "turn on" [14] FRET-based NO biosensor. In particular, while the absorption spectrum of CCP exhibits a characteristic high spin marker band at $645 \mathrm{~nm}\left(\varepsilon=3 \mathrm{mM}^{-1} \mathrm{~cm}^{-1}\right)$ [23], the binding of NO leads to the disappearance of this band [24] (see Fig. 1A). By attaching a fluorescent label to the protein, whose emission spectrum overlaps with the $645 \mathrm{~nm}$ band of the protein, this change in absorption upon NO binding can be translated into a change of fluorescence intensity of the label through a FRET mechanism. That is to say, when the protein is in the NO-free state, the label fluorescence becomes (partially) quenched as a result of FRET to the $645 \mathrm{~nm}$ band. As soon as NO binds to CcP, the latter band disappears and the energy absorbed by the label is emitted as fluorescence, thus causing an increase in fluorescence. In this way the fluorescent dye acts as a passive "beacon" which is "off" in the NO-free state and "on" in the NO-bound state of the protein. Two different labels, whose emission spectra overlap with the $645 \mathrm{~nm}$ spin marker band of $\mathrm{CcP}$, were selected for this study: Cy5 and Atto620. The overlap of the $645 \mathrm{~nm}$ high spin marker band of $\mathrm{CCP}$ with the emission spectrum of either Atto620 or Cy5 can be judged from Fig. 1B.

Several research groups have examined the interaction of CcP with small ligands other than NO (e.g., $\mathrm{CN}^{-}, \mathrm{F}^{-}$, and $\mathrm{CO}$ ) $[25,26]$. Some of the complexes formed upon interaction of CcP with small ligands were found not to be stable; for example the dioxygen adduct of $\mathrm{CcP}$ has a half life of $200 \mathrm{~ms}$ at $23{ }^{\circ} \mathrm{C}$ [27]. By contrast, NO is one of the most powerful strong field ligands, which occurs on the right end of the spectrochemical series [28,29]. One may expect, therefore, that the CcP-NO complex will exhibit excellent stability, which is clearly borne out when comparing, for example, the dissociation constant of the $\mathrm{CcP}^{-} \mathrm{F}^{-}$complex $(305 \mu \mathrm{M})$ [26] with that of the CcP-NO complex ( $10 \mu \mathrm{M}$, this work). This suggests that the binding of NO to CCP is strongly preferred over other analytes. This was a further reason to select CcP for implementation in an NO biosensor.

In this contribution we report on the use of CcP fluorescently labeled at the $\mathrm{N}$-terminus. We present data showing that the system is efficiently working in solution as a fluorescence-based NO sensor, allowing measurements in any kind of standard fluorescence measuring device. Furthermore we show that the sensing system operates also when labeled $\mathrm{CcP}$ is immobilized into a silica matrix, providing a more stable and, above all, reusable biosensor.

\section{Materials and methods}

\subsection{General}

$\mathrm{CcP}$ was prepared following literature procedures [22]. The purity of the $\mathrm{CcP}$ was checked spectrophotometrically by measuring the ratio of the absorbances at $408 \mathrm{~nm}$ and $280 \mathrm{~nm}$ or at $408 \mathrm{~nm}$ and $308 \mathrm{~nm}$ and comparing them with literature values $\left(\mathrm{A}_{408} / \mathrm{A}_{280}=1.28\right.$ or $\mathrm{A}_{408} /$ $\mathrm{A}_{308}=1.57$ ) [22]. Cy5 NHS-ester was purchased from Amersham Biosciences (Freiburg Germany) and Atto620 NHS-ester was purchased from ATTO-TEC Biolabeling and Ultraanalytics (Siegen, Germany). Stock solutions of the dyes $(50 \mathrm{mM})$ were prepared by dissolving the powders in water-free DMSO. TMOS (tetramethyl orthosilicate) was obtained from Sigma Aldrich. $\mathrm{NaNO}_{2}, \mathrm{FeSO}_{4} \cdot 7 \mathrm{H}_{2} \mathrm{O}$ and $\mathrm{H}_{2} \mathrm{SO}_{4}$ (37\% solution) were bought from Sigma Aldrich.

\subsection{Protein labeling}

CcP was labeled at the N-terminus with NHS-reactive fluorophors in a roughly 10 times molar excess over the protein concentration in potassium phosphate $100 \mathrm{mM}, \mathrm{pH} 6.8$, using procedures reported in the literature [30]. The constructs were purified using PD-10 gelfiltration columns purchased from Amersham Pharmacia Biotech. Labeling ratios were in the range of 0.2-0.9 (dye molecule/protein), as determined from the absorption spectra of the labeled proteins using the extinction coefficients at $280 \mathrm{~nm}$ [24] for the protein and at $620 \mathrm{~nm}$ for the Atto620 label and at $645 \mathrm{~nm}$ for the Cy5 label as stated by the manufacturers, respectively.
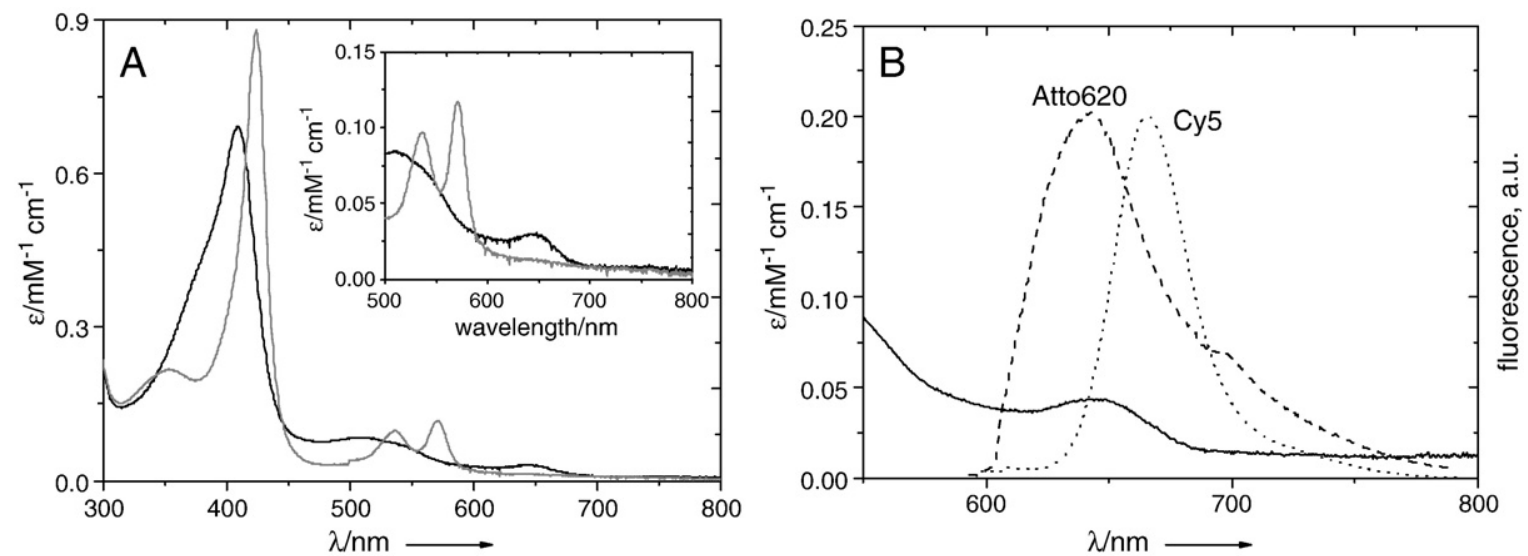

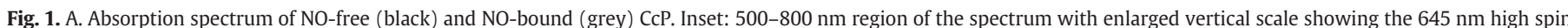

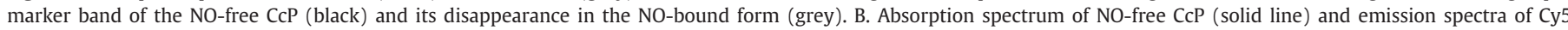

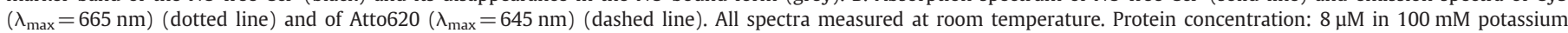
phosphate buffer $(\mathrm{pH}=6.8)$. 


\subsection{Protein encapsulation in TMOS sol-gel}

The preparation of silica gels and the encapsulation of proteins were undertaken with pure TMOS [31]. TMOS (15.22 g) was mixed with milliQ water $(3.38 \mathrm{~g}$ ) in a $1: 2$ molar ratio followed by the addition of $20 \mu \mathrm{l}$ of $10 \mathrm{mM} \mathrm{HCl}$. The reaction mixture was sonicated for $20 \mathrm{~min}$. Upon addition of buffer (potassium phosphate $100 \mathrm{mM}$, $\mathrm{pH} 6.8$ ) in a 1:1 (volume) ratio and addition of roughly $1 \mathrm{~mL}$ of labeled protein solution (end conc. of protein: $5-10 \mu \mathrm{M}$ ) the mixture was degassed by bubbling through pure argon. Before gelation $150 \mu \mathrm{L}$ of the sol solution was quickly poured onto a home made device $\left(8 \times 30 \mathrm{~mm}^{2}\right.$ quartz slide Heraeus 3 quality with a $1 \mathrm{~mm}$ thickness $)$ yielding a roughly $0.6 \mathrm{~mm}$ thick sol-gel layer on top of the quartz slide. Activation of quartz slides with "Piranha solution" $\left(30 \% \mathrm{H}_{2} \mathrm{O}_{2}\right.$ and concentrated $\mathrm{H}_{2} \mathrm{SO}_{4}$ in a 1:3 volume ratio) was performed before pouring the sol solution on top. [Caution: "Piranha solution" is highly corrosive and should be handled with extreme care]. Samples were kept sealed with parafilm and allowed to age at $4{ }^{\circ} \mathrm{C}$ overnight before use. Methanol, a by-product of the TMOS-based sol-gel process, was removed by washing the samples with potassium phosphate buffer (100 mM, pH 6.8) several times.

\subsection{Nitric oxide saturated solutions}

Saturated solutions of nitrogen monoxide were prepared by bubbling NO gas, produced in situ according to literature procedures [32], through $5 \mathrm{~mL}$ of $100 \mathrm{mM}$ potassium phosphate ( $\mathrm{pH} 6.8$ ) for periods of up to $1 \mathrm{~h}$ resulting in an approximate concentration of $2 \mathrm{mM}$ at $20^{\circ} \mathrm{C}[33]$.

\subsection{Absorbance and fluorescence measurements}

Absorption spectra were recorded on a Cary-50 Spectrophotometer. For the solution measurements fluorescence spectra and time traces were measured on a Cary Eclipse Spectrophotometer. For the sol-gel measurements fluorescence spectra and time traces were also measured on a Cary Eclipse Spectrophotometer by fixing the quartz slides with a home made device in a $10 \times 10 \mathrm{~mm}^{2}$ airtight quartz fluorescence cuvette (Hellma Benelux bv, Rijswijk, Netherlands) and applying front face illumination [34]. Measurements were performed in potassium phosphate buffer $(100 \mathrm{mM}, \mathrm{pH}$ $6.8)$ at room temperature. High quality argon $\left(<1 \mathrm{ppm} \mathrm{O}_{2}\right)$ was used to deoxygenate the sample prior to each measurement and to displace NO. For NO titrations, the cuvette was filled with deoxygenated sample solutions, after which $\mu \mathrm{L}$ amounts of an NOsaturated buffer were injected.

\subsection{Förster radius calculations}

Values for the Förster radius, $R_{0}$ were calculated from the equation [34]

$R_{0}=0.211\left(J \kappa^{2} n^{-4} \Phi_{D}\right)^{1 / 6}(\AA)$.

Here $k^{2}$ is an orientation factor, $n-$ refractive index, $\Phi_{\mathrm{D}}-$ fluorescence quantum yield of the donor and $J$ - spectral overlap integral, defined as $J=\int F_{\mathrm{D}}(\lambda) \varepsilon_{\mathrm{A}}(\lambda) \lambda^{4} d \lambda / \int F_{\mathrm{D}}(\lambda) d \lambda$, where $F_{\mathrm{D}}(\lambda)$ is the fluorescence intensity of the donor, $\varepsilon_{\mathrm{A}}(\lambda)$ - the extinction coefficient in $\left[\mathrm{M}^{-1} \mathrm{~cm}^{-1}\right]$ of the acceptor at wavelength $\lambda$ with $\lambda$ expressed in nanometers. The refractive index was taken as 1.4 and the orientation factor $\kappa^{2}$ was taken as $2 / 3[34,35]$. $\Phi_{\mathrm{D}}$ for Cy5 was taken as 0.27 [36], for Atto620 as 0.5 (as stated by the manufacturers). The distance $(R)$ from the Cy5 and Atto620 labels to the active site was estimated as $R=[(d+1) \pm 0.5] \mathrm{nm}$ (adding $1 \mathrm{~nm}$ to the calculated distance $d$ accounts for the approximate length of the linker chain), where $d$ is the distance from the attachment point of the dye (N-terminus) to the Fe atom in the heme. The distance $d$ was estimated at $3.0 \mathrm{~nm}$ from the crystal structure of CcP from the baker's yeast [1ZBY].

\subsection{Nano-HPLC-ESI-MS analysis}

For the determination of molecular masses samples were analyzed using a Q-TOF instrument, QSTAR Elite (Applied Biosystems, Foster City, $\mathrm{CA} /$ Toronto, Canada) equipped with a nanoflow electrospray ion source. Pulled silica capillary ( $170 \mu \mathrm{m}$ OD/100 $\mu \mathrm{m}$ ID, tip $30 \mu \mathrm{m}$ ID) was used as nanoflow tip. The samples $(15 \mathrm{pmol})$ were loaded, purified and concentrated on a pre-column PepMap, C18, $5 \mathrm{~mm}$ length, $300 \AA$, (LCPackings, Sunnyvale, CA USA) at $30 \mu \mathrm{L} / \mathrm{min}$ flow rate. HPLC analysis was performed by Ultimate 3000 (Dionex, Sunnyvale, CA USA) using a capillary column, PepMap, C18, 15 cm length, $75 \mu \mathrm{m}$ ID, 300 Å (LCPackings, Sunnyvale, CA USA), solvent A: $2 \%$ ACN in $0.1 \%$ formic acid ( $\mathrm{HCOOH})$ and $0.025 \%$ TFA and solvent $\mathrm{B}: 98 \% \mathrm{ACN}$ in $0.1 \% \mathrm{HCOOH}$ and $0.025 \% \mathrm{TFA}$ at $300 \mathrm{~nL} / \mathrm{min}$ flow rate. The following gradient was used: $5-50 \% \mathrm{~B}$ in $30 \mathrm{~min}, 50-98 \% \mathrm{~B}$ in $6 \mathrm{~s}$. Mass spectra were acquired in the mass range of $\mathrm{m} / \mathrm{z} 300-2000$ in positive ion mode. Molecular mass measurements were performed on the top 20 scans of the chromatographically eluted proteins. Analyst QS.2.0 software was used for data acquisition and Bioanalyst QS 2.0 for Bayasian protein reconstruct. Two independent nano-HPLC-ESI-MS experiments were performed for each sample.

\section{Results and discussion}

\subsection{Fluorescence spectroscopy}

CcP was labeled either with Atto620 or with Cy5. The pH of the labeling reaction was chosen so as to favour labeling of the $\mathrm{N}$ terminus over lysine labeling (see Materials and methods). These conditions have been tested for the case of azurin, for which it was confirmed by electrospray MS that the label was exclusively present on the N-terminus [30]. In the present case concentrations were chosen to ensure that the dye-to-protein ratio was less than 1 . While a small dye-to-protein ratio affects only the sensitivity of the experiment, a ratio larger than 1 leads to ambiguities in donor-acceptor distances between the attached label and the prosthetic group of the protein. Moreover, it has been found that proteins labeled with multiple amino-reactive labels show a decrease in fluorescence due to mutual resonance energy transfer [37]. To check the number of label molecules per $\mathrm{CcP}$, electrospray ionization mass spectrometry was performed on different Atto620 labeled CcP samples. Only peaks arising from unlabeled and singly labeled proteins were observed, showing that no $\mathrm{CcP}$ molecules with multiple labels were present in the samples (see Fig. S1).

To test the system, the fluorescence intensity of N-terminally labeled CcP was monitored as a function of time during a change from an NO-saturated to an NO-free environment. Fig. 2 shows a typical time trace of a solution containing $140 \mathrm{nM}$ of dye-labeled CcP when excited at the absorption maximum $(\lambda=619 \mathrm{~nm})$ of the dye (Atto620). In this particular experiment each cycle was started by adding NO to an end concentration of $360 \mu \mathrm{M}$ (i.e., in considerable excess over the CcP concentration) and completed by passing argon through the solution for the complete removal of NO. The dye emission was followed at $640 \mathrm{~nm}$.

A fast increase in label emission was clearly observed upon each NO addition. When bubbling through argon to displace the NO, the fluorescence intensity of the label diminished again; the cycle could be repeated many times. This finding showed that the NO binding process is reversible, which is crucial for practical sensing applications. The dye switching ratio (SR) defined by

$S R \equiv\left(F_{\mathrm{CCP}-\mathrm{NO}}-F_{\mathrm{CCP}}\right) / F_{\mathrm{CCP}-\mathrm{NO}}$ 


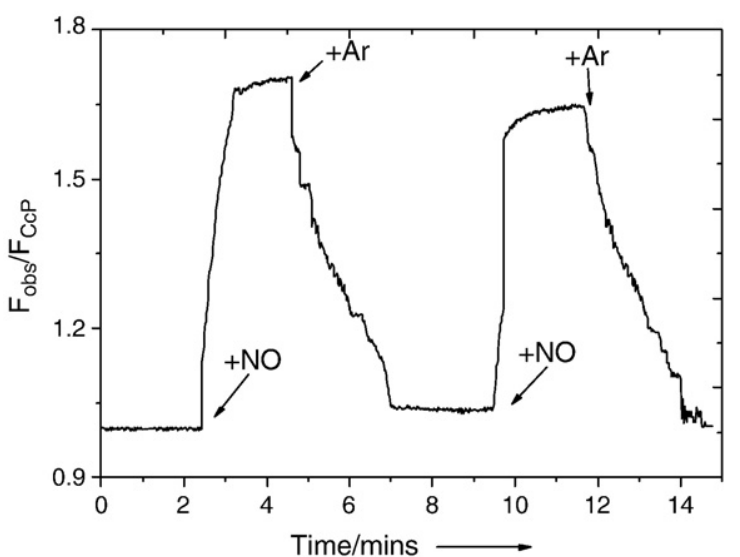

Fig. 2. Room temperature fluorescence intensity time trace observed at $645 \mathrm{~nm}$ (exc $619 \mathrm{~nm}$ ) of Atto620 labeled CCP, upon addition and removal of NO. The noise in the experimental trace after starting the bubbling of argon is due to the perturbation of the optical path of the measuring beam by the bubbling. Protein concentration: $140 \mathrm{nM}$ in $100 \mathrm{mM}$ potassium phosphate buffer $(\mathrm{pH}=6.8)$.

in which $F_{\mathrm{CCP}-\mathrm{NO}}$ and $F_{\mathrm{CCP}}$ are the emission intensities of the NO-bound and NO-free protein, is $45 \pm 5 \%$. This can be compared with the theoretical value which is given by

$S R \equiv[E(C c P)-E(C c P-N O)] /[1-E(C c P-N O)]$

where $E(\mathrm{CcP})$ and $E(\mathrm{CcP}-\mathrm{NO})$ are the theoretical FRET efficiencies in the NO-free and NO-bound state of CCP. $E$ is given by $E=R_{0}^{6} /\left(R_{0}^{6}+R^{6}\right)$ [34] where $R_{0}$ is the Förster radius and $R$ the distance between donor and acceptor. In the present case, the estimated Förster radii for FRET from Atto620 to the heme (see Materials and methods) are $4.2 \mathrm{~nm}$ for the NO-free and $3.6 \mathrm{~nm}$ for the NO-bound state of CcP, while the donor-acceptor distance from Atto620 to the heme is $3.0 \mathrm{~nm}$ (as estimated from the crystal structure of CcP from baker's yeast [1ZBY], see also Fig. 3).

This leads to theoretical transfer efficiencies of 0.88 for the NO-free state and 0.75 for the NO-bound state of CCP and a theoretical SR of $52 \%$. Considering the uncertainties in the orientation factor, $\kappa$, (see Eq. (1)) and in the distance $R$, this value is in good agreement with the experimental value of $45 \pm 5 \%$.

It was found that the maximum and minimum fluorescence levels slowly decrease with the number of cycles the sample solution had gone through. This could be due to partial denaturation of the $\mathrm{CcP}$ after bubbling argon through the solution to displace the NO. In fact the fluorescence intensity of the construct after adding NO remained constant for hours when the solution was left standing. Under the same conditions, Cy5 labeled CCP exhibited a SR of $40 \pm 5 \%$ (data not shown). In this case the theoretically estimated $R_{0}$ values are $3.8 \mathrm{~nm}$ for the NO-free state and $3.3 \mathrm{~nm}$ for the NO-bound state and a theoretical SR of 56\%. These experiments have been performed at $\mathrm{pH}$ 6.8 and provide a proof-of-principle for the proposed methodology. Additional studies, to be reported elsewhere, will be undertaken to validate the method for a range of $\mathrm{pH}$ values.

\subsection{Effect of anions and oxygen}

The fluorescence intensity of the Atto620 labeled CcP system upon $\mathrm{NO}$ binding in the presence of large excesses of $\mathrm{NO}_{2}^{-}, \mathrm{NO}_{3}^{-}$, examples of biologically relevant and potentially competing ions, was checked to obtain an indication on the selectivity of the construct. It is evident from Fig. 4 that in the presence of a large excess of $\mathrm{NO}_{2}^{-}$or $\mathrm{NO}_{3}^{-}$over the protein concentration there is still a clear and large effect on the sample fluorescence when $\mathrm{NO}$ is introduced into the solution. $\mathrm{NO}_{2}^{-}$ causes a slight increase in the fluorescence (see Fig. 4). It is known

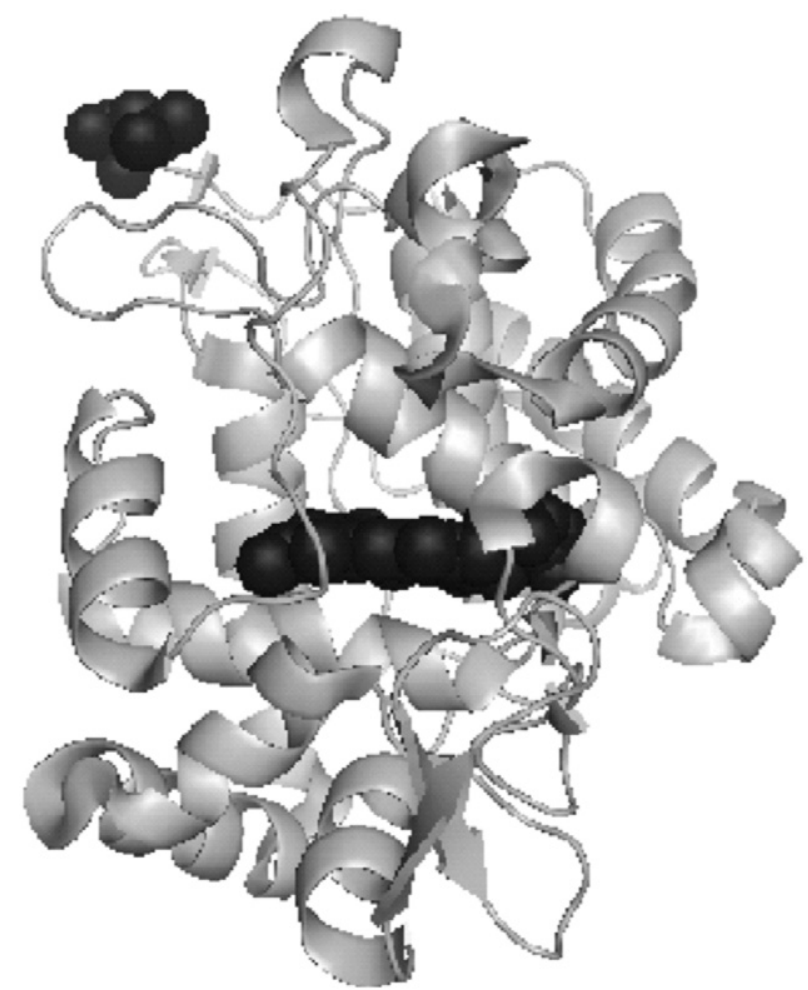

Fig. 3. Structure of $\mathrm{CCP}$ showing the heme (black spheres) and Leu1 (black). Leu1 corresponds to the attachment point of the label. The structure has been obtained from the PYMOL programme using the reported X-ray diffraction structure of CcP (PDB 1ZBY).

that $\mathrm{NO}_{2}^{-}$under acidic conditions is partly converted into $\mathrm{NO}$ and the observed increase in fluorescence is ascribed to this effect.

To assess the potential of the NO sensing system in the presence of oxygen, the fluorescence intensity of CcP labeled with Atto 620 was monitored when saturating the buffer with oxygen prior to the measurements. A fast and sizeable increase in the fluorescence intensity upon NO addition is observed (Fig. S2). This shows that the rate of NO binding to $\mathrm{CCP}$ is fast compared with the rate of reaction of $\mathrm{NO}$ with $\mathrm{O}_{2}$, and that on the time scale of the measurement $\mathrm{O}_{2}$ does not interfere with NO detection.

\subsection{Determination of $K_{d}$}

To determine the binding affinity of $\mathrm{CcP}$ for NO, NO titrations of labeled $\mathrm{CcP}$ were performed while monitoring the fluorescence

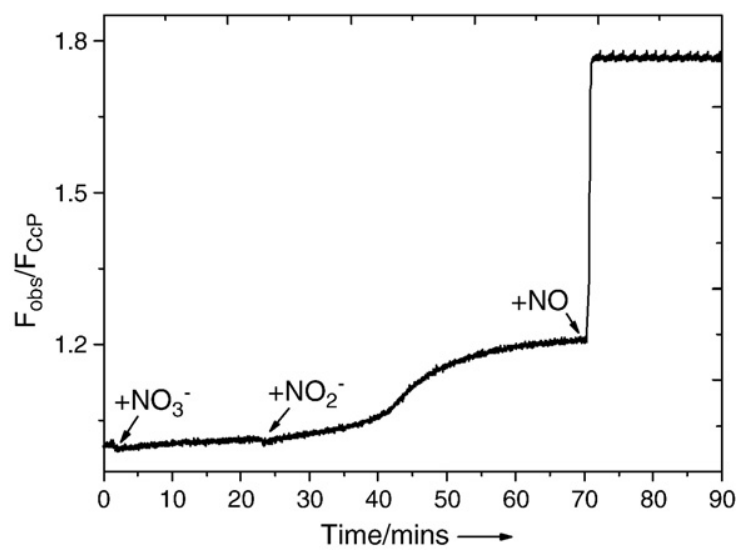

Fig. 4. Fluorescence time trace of Atto620 labeled $\mathrm{CcP}$ after subsequent additions of $360 \mu \mathrm{M}$ of $\mathrm{NO}_{3}^{-}, 360 \mu \mathrm{M}$ of $\mathrm{NO}_{2}^{-}$and $360 \mu \mathrm{M}$ of NO. Protein concentration: $60 \mathrm{nM}$ in $100 \mathrm{mM}$ potassium phosphate buffer $(\mathrm{pH}=6.8)$. 
intensity of the labels (Figs. S3, S4). The relation between the observed fluorescence intensity, $F_{\mathrm{NO}}$, and $K_{\mathrm{d}}$ is given by [20]:

$F_{\mathrm{NO}}=F_{0}-\left\{\left(F_{0}-F_{\infty}\right) \cdot[\mathrm{NO}]\right\} /\left\{[\mathrm{NO}]+K_{\mathrm{d}}\right\}$

where [NO] is the concentration of free NO in solution and $F_{0}$ and $F_{\infty}$ denote the emission intensities of the NO-free and NO-bound protein, respectively. According to Eq. (4) $K_{\mathrm{d}}$ is independent of the labeling ratio. A different labeling ratio is equivalent to introducing a scale factor for the fluorescence intensities which leaves Eq. (4) invariant.

Since the protein concentrations used in the experiments were small compared to the NO concentrations, for practical purposes the free [NO] could be set equal to the total amount of added NO and the data points in Figs. S3, S4 could be directly fitted to Eq. (4) resulting in a $K_{\mathrm{d}}$ of $9.4 \pm 0.5 \mu \mathrm{M}$ for the Atto620 CcP system and of $12.0 \pm 2 \mu \mathrm{M}$ for the $\mathrm{Cy} 5 \mathrm{CcP}$ construct. While we could not find literature data on the $K_{\mathrm{d}}$ for NO, the $K_{\mathrm{d}}$ values found here are in mutual agreement.

\subsection{Determination of NO binding kinetics}

To assess the NO binding kinetics, we focused on the Atto620 CcP system. The fluorescence of the labeled CcP was measured as a function of time after adding various amounts of $\mathrm{NO}$ in the range of 1-75 $\mu \mathrm{M}$. The fluorescence time traces (see Fig. S5 for examples) could be well fitted to an exponential function

$F_{\mathrm{obs}}=F_{0}+\left\{F(t=\infty)-F_{0}\right\}\left\{1-\exp \left(-k_{\mathrm{obs}} \cdot t\right)\right\}$.

When plotting the $k_{\mathrm{obs}}$ values as a function of the NO concentration, a linear dependence was observed. As an example, in Fig. 5 the data are shown that were observed on a sample containing $20 \mathrm{nM}$ of Atto620 labeled CcP. When using for $k_{\text {obs }}$ the expression $k_{\text {obs }}=k_{\text {off }}+$ $k_{\text {on }}[\mathrm{NO}]$ with $k_{\text {off }}$ the rate of dissociation of the NO from the $\mathrm{CcP} / \mathrm{NO}$ complex and $k_{\text {on }}$ the second order rate constant for the association of $\mathrm{NO}$ and $\mathrm{CCP}$, the following values could be extracted from the data in Fig. 5: $k_{\text {off }}=0.22 \pm 0.08 \mathrm{~min}^{-1}$, and $k_{\text {on }}=0.024 \pm 0.002 \mu \mathrm{M}^{-1} \mathrm{~min}^{-1}$. From these a value of $K_{\mathrm{d}}=k_{\text {off }} / k_{\text {on }}=9 \pm 3 \mu \mathrm{M}$ is found in agreement with the $K_{\mathrm{d}}$-values derived from the NO titrations (see above).

$K_{\mathrm{d}}$ is the key parameter for determining the sensitivity of the sensor. $K_{d}$ is defined as $[\mathrm{CCP}][\mathrm{NO}] /[\mathrm{CCP}-\mathrm{NO}]$ where $[\mathrm{CcP}]$ is the concentration of the NO-free and $[\mathrm{CCP}-\mathrm{NO}]$ the concentration of the NO-bound CcP. The quantity by which the sensitivity of the method can be judged is the ratio $[\mathrm{CCP}] /[\mathrm{CcP}-\mathrm{NO}]$. This ratio varies strongly when the [NO] is close to the $K_{\mathrm{d}}$. Therefore, with the proposed biosensing system, NO can be easily detected and quantified in the concentration range of between $1 \mu \mathrm{M}$ and $100 \mu \mathrm{M}$ with simple fluorescence detection. For instance, with activated macrophages

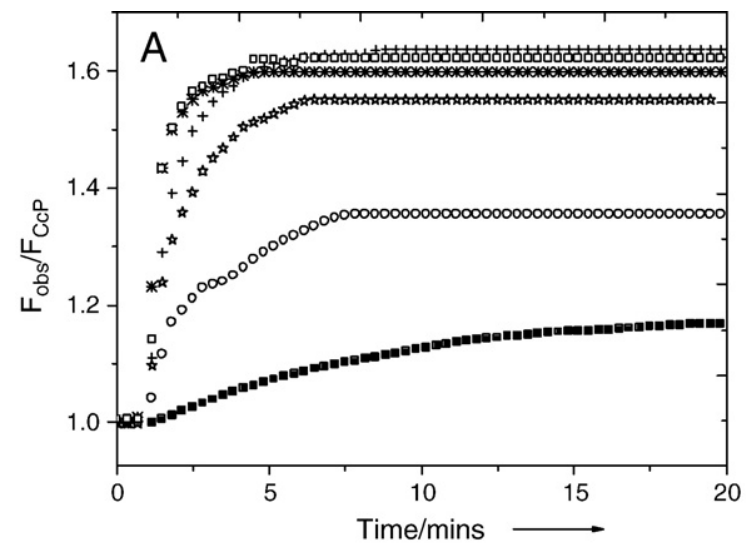

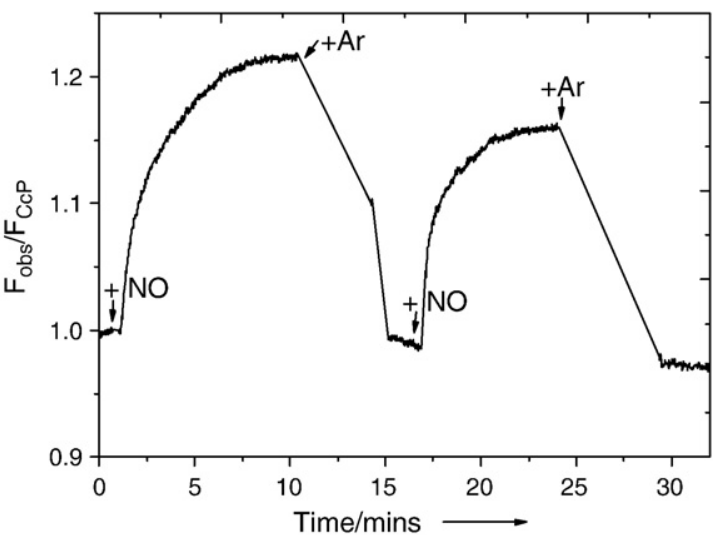

Fig. 6. Fluorescence intensity time trace at $665 \mathrm{~nm}$ (exc $645 \mathrm{~nm}$ ) of Cy5-labeled CcP entrapped into a TMOS-based sol-gel immobilized on a quartz support upon addition and removal of NO. During the measurement the sensing device was placed in $100 \mathrm{mM}$ potassium phosphate buffer $(\mathrm{pH}=6.8)$ at room temperature. The fluorescence was recorded while alternatingly NO was added (to a final concentration of $360 \mu \mathrm{M}$ ) to the solution and removed by refreshing the buffer and bubbling argon through the solution.

the transient NO concentration in cells or tissue is in the micromolar range $[17,18]$. The sensor described here would be suitable, therefore, for detecting extracellular macrophage derived NO. Future work will address this topic. Other examples relate to monitoring of industrial exhaust gases where NO levels may rise into the micromolar range $[38,39]$, the study of the physiological effects of elevated NO levels in mammals [40,41], and the study of the equilibrium kinetics of transition metal complexes in which $\mathrm{NO}$ is one of the coordinating ligands $[42,43]$.

\subsection{Immobilization into sol-gel matrices}

In order to investigate the applicability of our protein-based method for implementation in a solid state NO sensing device, we immobilized fluorescently labeled CCP in a silica (TMOS) matrix [44]. CcP N-terminally labeled with either Cy5 or Atto620 was entrapped in TMOS and immobilized on a quartz support as described in "Materials and methods". Binding of NO to the immobilized and entrapped CcP was found to be reversible: insertion of the construct into an NO containing solution resulted in a fluorescence increase. Subsequent bubbling of argon through the solution removed the NO again (Fig. 6). Labeled CcP immobilized into a TMOS matrix kept its activity in terms of SR and time response for more than 20 days when stored at $4{ }^{\circ} \mathrm{C}$.

The SRs observed were lower than the ones obtained in the solution measurements (e.g., in the case of $360 \mu \mathrm{M}$ NO an SR of $20 \%$ was found

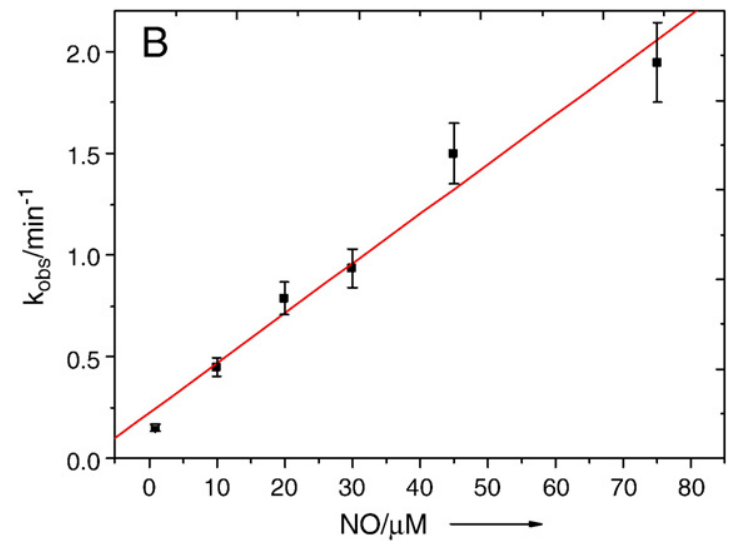

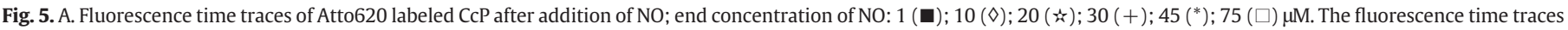

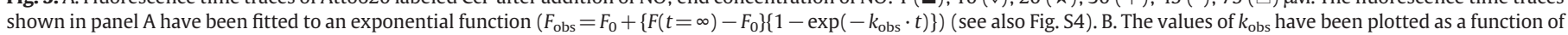
the NO concentration. Protein concentration: $20 \mathrm{nM}$ in $100 \mathrm{mM}$ potassium phosphate buffer $(\mathrm{pH}=6.8)$. All time traces were measured at room temperature. 
with Cy5-labeled CcP when measuring the sol-gel sample, while $40 \pm$ $5 \%$ was found in the bulk experiment). These findings may be related to a fraction of encapsulated protein not being accessible to the substrate or having been damaged and having lost its capability to bind NO. The fluorescence time traces could be fitted to the same exponential function as used for the solution experiments (see Eq. (5)).

Not surprisingly, the rates of NO binding were smaller, most likely due to the time needed for the NO to diffuse into the TMOS matrix. A similar behaviour was found for immobilized hemocyanin when it was used to monitor oxygen concentrations [44].

\subsection{Controls}

No significant changes in the fluorescence intensities were observed in the absence of NO or when free dyes, Cy5 or Atto620 labeled BSA, or unlabeled $\mathrm{CCP}$ were used as sensing materials. Furthermore when adding $360 \mu \mathrm{M}$ of nitrate, $\mathrm{CO}$ and oxygen, the proposed sensor did not exhibit a change in fluorescence (Fig. S6). The lack of an effect of $\mathrm{CO}$ and $\mathrm{O}_{2}$ binding is most likely due to the fact that the $\mathrm{CcP}$ we are working with is in the $\mathrm{Fe}^{3+}$ state while $\mathrm{O}_{2}$ and $\mathrm{CO}$ have a strong preference for $\mathrm{Fe}^{2+}$. Differently, when adding $360 \mu \mathrm{M}$ of nitrite, the fluorescence intensity of the system slightly increased. As argued above, this is probably due to the NO produced by nitrite (Fig. S6).

\section{Conclusions}

Fluorescently labeled CcP can be successfully used as a 'turn on' [14] FRET-based biosensor for NO. Its flexible mode of operation not only in a solution but also when applied in the form of a solid state device, makes a CcP-based device suited for sensing applications. A limitation is that the maximum and minimum fluorescence levels decrease with the number of cycles in a single experiment. Further experiments are needed to design a proper calibration scheme to overcome this limitation.

Our NO sensing system is based on the appearance of a fluorescence signal upon NO binding different from the already existing protein-based methods for NO detection [16,17,45]. Although concentration-dependent quenching mechanisms have shown to be successful, $[16,20]$ they are inherently less sensitive than methods exploiting fluorescence enhancement or "turn on" as a result of binding $[12,14,46]$. Moreover, it is often difficult to distinguish analyte response from sensor degradation when quenching is relied upon for quantitation [46]. The successful immobilization of labeled CcP into a polymeric matrix to provide a solid state NO sensing device presents an advantage in terms of reusability and stability of the system.

$\begin{array}{ll}\text { List of abbreviations } \\ \text { NO } & \text { nitric oxide } \\ \text { FRET } & \text { Förster Resonance Energy Transfer } \\ \text { DAN } & \text { o-diaminonaphthalene } \\ \text { DAF } & \text { o-diamino-fluorescein } \\ \text { Cytc } & \text { cytochrome } c^{\prime} \\ \text { CcP } & \text { cytochrome } c \text { peroxidase from baker's yeast } \\ \text { NHS } & \text { N-hydroxy-succinimide } \\ \text { TMO } & \text { tetramethyl orthosilicate } \\ \text { ESI-MS } & \text { electrospray ionization mass spectrometry } \\ \text { TFA } & \text { trifluoroacetic acid } \\ \text { ACN } & \text { acetonitrile }\end{array}$

\section{Acknowledgements}

We acknowledge Dr. Armand W.J.W. Tepper (University of Leiden, LIC) for helpful discussions and assistance with the preparation of the figures, Lionel Ndamba (University of Leiden, LIC) for assistance during protein purification, Dr. Gabriella Pocsfalvi (Istituto di Biochimica delle Proteine, CNR Naples) for her help with ESI mass spectrometry analysis.

\section{Appendix A. Supplementary data}

Supplementary data associated with this article can be found, in the online version, at doi:10.1016/j.jinorgbio.2010.02.002.

\section{References}

[1] D.E. Koshland Jr., Science 258 (1992) 1861.

[2] E. Culotta, D.E. Koshland Jr., Science 258 (1992) 1862-1865.

[3] S. Moncada, R.M. Palmer, E.A. Higgs, Pharmacol. Rev. 43 (1991) 109-142.

[4] L.L. Thomsen, D.W. Miles, Cancer Metastasis Rev. 17 (1998) 107-118.

[5] P.K. Lala, Cancer Metastasis Rev. 17 (1998) 1-6.

[6] J.C. Ball, M.D. Hurley, A.M. Straccia, C.A. Gierczak, Environ. Sci. Technol. 33 (1999) 1175-1178.

[7] G. Dooly, C. Fitzpatrick, E. Lewis, Energy 33 (2007) 657-666.

[8] E.M. Boon, M.A. Marletta, J. Am. Chem. Soc. 128 (2006) 10022-10023.

[9] K. Kikuchi, T. Nagano, H. Hayakawa, Y. Hirata, M. Hirobe, Anal. Chem. 65 (1993) 1794-1799.

[10] L. Mao, G. Shi, Y. Tian, H. Liu, L. Jin, K. Yamamoto, S. Tao, J. Jin, Talanta 46 (1998) 1547-1556.

[11] Y. Kotake, T. Tanigawa, M. Tanigawa, I. Ueno, D.R. Allen, C.S. Lai, Biochim. Biophys Acta 1289 (1996) 362-368.

[12] M.H. Lim, S.J. Lippard, Inorg. Chem. 43 (2004) 6366-6370.

[13] T. Nagano, T. Yoshimura, Chem. Rev. 102 (2002) 1235-1270.

[14] M.H. Lim, S.J. Lippard, Acc. Chem. Res. 40 (2007) 41-51.

[15] K.J. Franz, N. Singh, B. Spingler, S.J. Lippard, Inorg. Chem. 39 (2000) 4081-4092.

[16] S.L. Barker, R. Kopelman, T.E. Meyer, M.A. Cusanovich, Anal. Chem. 70 (1998) 971-976.

[17] S.L. Barker, H.A. Clark, S.F. Swallen, R. Kopelman, A.W. Tsang, J.A. Swanson, Anal Chem. 71 (1999) 1767-1772.

[18] S.L. Barker, Y. Zhao, M.A. Marletta, R. Kopelman, Anal. Chem. 71 (1999) 2071-2075.

[19] M. Sato, N. Hida, Y. Umezawa, Proc. Natl. Acad. Sci. U. S. A 102 (2005) $14515-14520$

[20] G. Zauner, E. Lonardi, L. Bubacco, T.J. Aartsma, G.W. Canters, A.W. Tepper Chemistry 13 (2007) 7085-7090.

[21] W. Erker, S. Sdorra, T. Basche, J. Am. Chem. Soc. 127 (2005) 14532-14533.

[22] J.A. Worrall, U. Kolczak, G.W. Canters, M. Ubbink, Biochemistry 40 (2001) 7069-7076.

[23] A.E. Pond, M. Sono, E.A. Elenkova, D.E. McRee, D.B. Goodin, A.M. English, J.H. Dawson, J. Inorg. Biochem. 76 (1999) 165-174.

[24] T. Yonetani, H. Yamamoto, J.E. Erman, J.S. Leigh Jr., G.H. Reed, J. Biol. Chem. 247 (1972) 2447-2455.

[25] S.L. Edwards, T.L. Poulos, J. Biol. Chem. 265 (1990) 2588-2595.

[26] T. Yonetani, H. Anni, J. Biol. Chem. 262 (1987) 9547-9554.

[27] M.A. Miller, A. Shaw, J. Kraut, Nat. Struct. Biol. 1 (1994) 524-531.

[28] R. Tsushida, Bull. Soc. Japan (1938) 388-400.

[29] C.K. Jorgensen, Absorption Spectra and Chemical Bonding in Complexes, Pergamon Press, Oxford, 1962.

[30] S. Kuznetsova, G. Zauner, R. Schmauder, O.A. Mayboroda, A.M. Deelder, T.J. Aartsma, G.W. Canters, Anal. Biochem. 350 (2006) 52-60.

[31] L.M. Ellerby, C.R. Nishida, F. Nishida, S.A. Yamanaka, B. Dunn, J.S. Valentine, J.I. Zink, Science 255 (1992) 1113-1115.

[32] B. Mattson, M. Anderson, S. Mattson, Microscale Gas Chemistry Book Educational Innovations, , 2003.

[33] G.W.C. Kaye, T.H. Laby, Tables of Physical and Chemical Constants, Longmans, London, 1966

[34] J.R. Lakovicz, Principles of Fluorescence Spectroscopy, Springer, 1996.

[35] L. Stryer, R.P. Haugland, Proc. Natl. Acad. Sci. U.S.A 58 (1967) 719-726.

[36] R.B. Mujumdar, L.A. Ernst, S.R. Mujumdar, C.J. Lewis, A.S. Waggoner, Bioconjug Chem. 4 (1993) 105-111.

[37] H.J. Gruber, C.D. Hahn, G. Kada, C.K. Riener, G.S. Harms, W. Ahrer, T.G. Dax, H.G Knaus, Bioconjug. Chem. 11 (2000) 696-704.

[38] E. Hawe, G. Dooly, C. Fitzpatrick, P. Chambers, E. Lewis, W.Z. Zhao, T. Sun, K.T.V Grattan, M. Degner, H. Ewald, S. Lochmann, G. Bramman, C. Wei, D. Hitchen, J. Lucas, A. Al-Shamma'a, E. Merlone-Borla, P. Faraldi, M. Pidria, Int. J. Intell. Syst. Technol. Appl. 3 (2007) 33-51.

[39] G. Dooly, C. Fitzpatrick, E. Lewis, J. Phys. Conf. Ser. 76 (012021) (2007).

[40] O.T. Bowen, G.F. Erf, M.E. Chapman, R.F. Wideman Jr., Poult. Sci. 86 (2007) 2550-2554.

[41] M.E. Chapman, R.F. Wideman Jr., Poult. Sci. 85 (2006) 312-320

[42] J.N. Bates, M.T. Baker, R. Guerra Jr., D.G. Harrison, Biochem. Pharmacol. 42 (1991) S157-S165.

[43] F. Roncaroli, J.A. Olabe, R. Van Eldik, Inorg. Chem. 42 (2003) 4179-4189.

[44] G. Zauner, M. Strianese, L. Bubacco, T.J. Aartsma, A.W. Tepper, G.W. Canters, Inorg. Chim. Acta 361 (2008) 1116-1121.

[45] S.L. Barker, R. Kopelman, Anal. Chem. 70 (1998) 4902-4906.

[46] B.R. White, H.M. Liljestrand, J.A. Holcombe, Analyst 133 (2008) 65-70. 